\title{
Camallanus ctenopomae n. sp. (Nematoda; Camallanidae), parasite de Ctenopoma kingsleyae Gunther (Poisson ; Anabantidae) au Sénégal
}

\author{
par G. VASSILIADES et A. J. PETTER \\ Laboratoire national de recherches vétérinaires, Dakar, Sénégal, \\ Institut d'Elevage et de Médecine vétérinaire des Pays tropicaux, F 94700 Maisons Alfort \\ et Laboratoire de Zoologie (Vers) associé au C.N.R.S. ( $\mathrm{P}^{\mathrm{r}}$ A.-G. ChaBaUd), \\ Muséum national d'Histoire naturelle, 57, rue Cuvier, F 75005 Paris
}

\begin{abstract}
Résumé
Description d'un nouveau Camallanidae; Camallanus cteno. pomae n. sp. parasite de Ctenopoma kingsleyae Poisson Anabantidae du Sénégal. Cette espèce diffère des autres Camallanus par les caractères de sa capsule buccale, de ses spicules et de ses extrémités caudales mâle et femelle.
\end{abstract}

\section{Summary}

A new species is described: Camallanus ctenopomae n. sp. (Camallanidae) parasite of Ctenopoma kingsleyae (Anabantidae Fish) in Senegal. This species is characterized by its buccal capsule, its spicules and the tails of both male and female.

A l'occasion de l'autopsie d'un poisson Anabantidae (Ctenopoma kingsleyae) pêché dans un marigot de Sangalkam, près de Rufisque, nous avons pu récolter quelques Nématodes parasites appartenant au genre Camallanus Railliet et Henry, 1915. Nous en donnons ci-après la description. 


\section{Matériel étudié :}

Hôte: Ctenopoma kingsleyae Günther, 1896. Famille des Anabantidae, sousordre des Anabantoidei, Perciformes, Téléostéens.

Lieu de récolte : marigot de Sangalkam, près de Rufisque (Région du Cap Vert), Sénégal, le 27 avril 1970.

Localisation : intestin moyen.

Matériel récolté : 5 mâles et 2 femelles, mis en collection au Laboratoire d'Helminthologie du Laboratoire national de recherches vétérinaires de Dakar sous le numéro A 61. Types : 1 mâle et 1 femelle déposés dans les collections du Muséum national d'Histoire naturelle de Paris (Laboratoire de Zoologie - Vers) sous le numéro 170 B A.

\section{Description :}

\section{GÉNÉRALITÉs.}

Les nématodes étudiés sont de taille moyenne, la femelle mûre étant généralement 2 fois plus grande que le mâle. Leur corps est brunâtre, d'aspect trapu, peu aminci à ses extrémités. La cuticule est finement striée, mince.

Ils présentent une capsule buccale à parois épaisses constituée par 2 valves latérales et pourvue de 2 tridents médians.

La bouche est allongée dorso-ventralement, elle est entourée par 4 papilles submédianes grosses et saillantes et 2 petites amphides latérales peu apparentes (cf. fig. I, D).

L'œsophage est divisé en 2 parties, un œsophage musculaire antérieur légèrement renflé à sa base et soutenu intérieurement par 3 baguettes chitinoïdes longitudinales, suivi d'un œsophage glandulaire élargi en massue postérieurement et 2 fois plus long que l'œsophage musculaire.

L'anneau nerveux est situé au niveau du quart antérieur de l'œsophage musculaire ; un peu en arrière il existe 2 deirides minuscules; le pore excréteur se situe à mi-distance entre l'anneau nerveux et la jonction des 2 œsophages.

La femelle est vivipare, à vulve légèrement pré-équatoriale; le mâle présente 2 spicules inégaux.

\section{DESCRIPTION DE LA RÉGION BUCCALE.}

La capsule buccale est limitée par 2 valves latérales articulées, à parois très épaisses. La surface de chaque valve présente 9 baguettes chitinoïdes longitudinales parallèles, armées de nombreuses dents sur toute leur longueur, donnant à ces baguettes un aspect denticulé ; et 2 petites pièces chitinoïdes triangulaires situées antérieurement (cf. fig. I, B).

A la jonction des valves, dans le plan médian, dorsalement et ventralement, la capsule buccale porte également 2 grosses pièces chitinoïdes en forme de trident dont les pointes sont dirigées vers l'arrière du corps (cf. fig. I, C). 

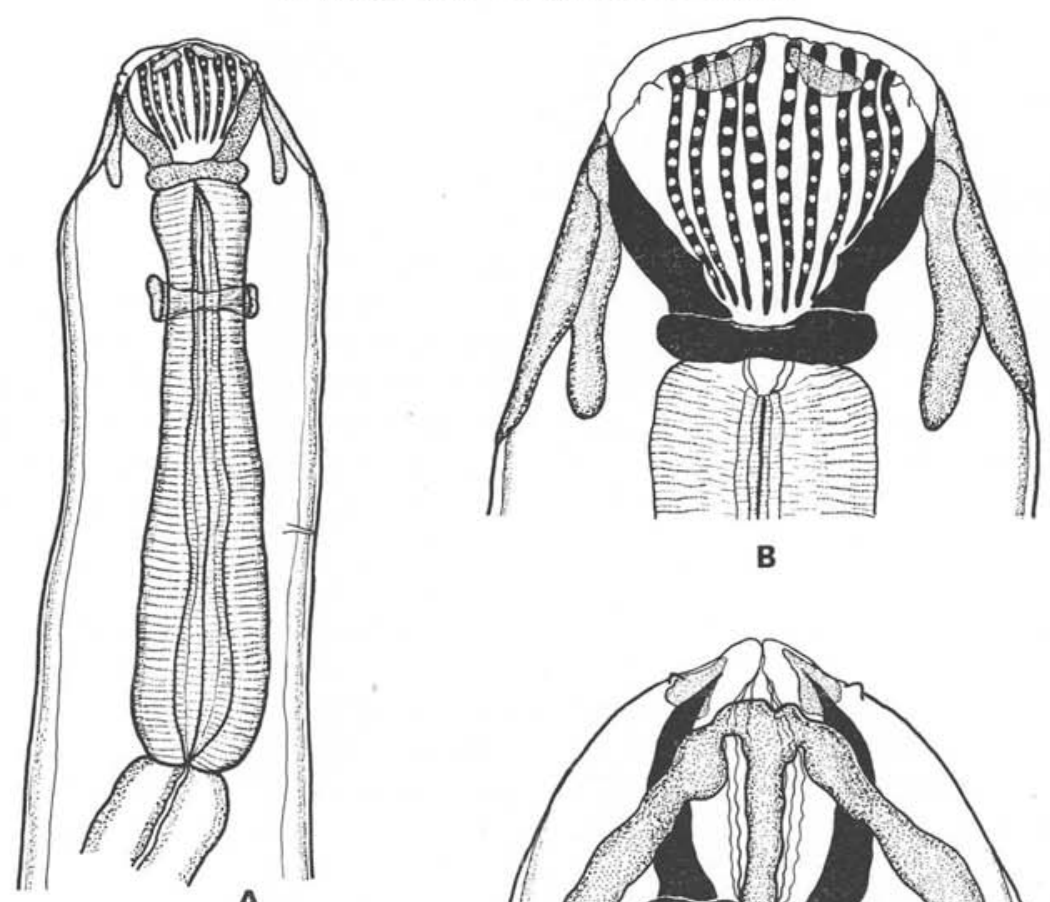

B
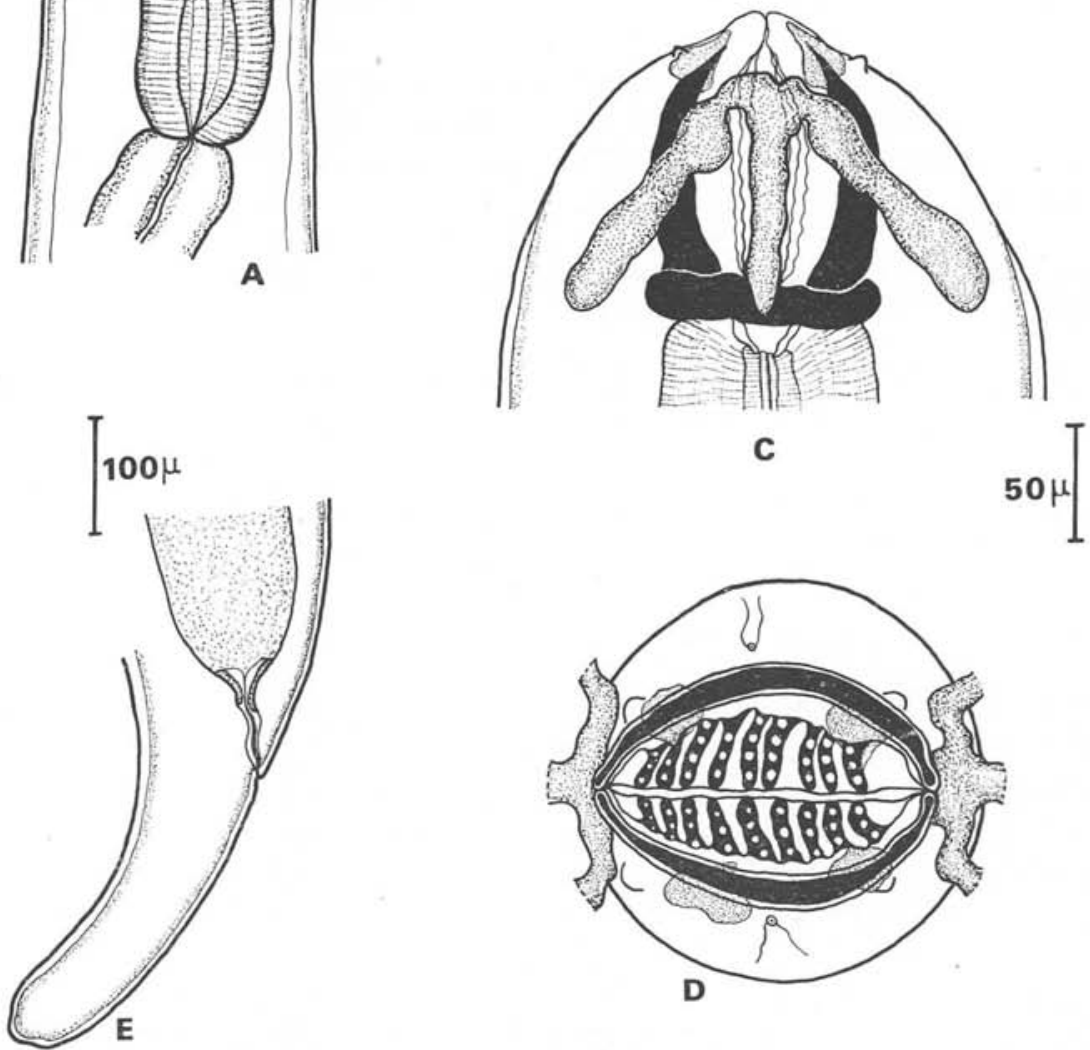

Fig. 1. - Camallanus ctenopomae n. sp. A : région antérieure, mâle, vue latérale. B: capsule buccale, femelle, vue latérale. C: capsule buccale, femelle, vue médiane. D: capsule buccale, femelle, vue apicale. B: queue de la femelle, vue latérale (échelle $50 \mu: \mathrm{B}, \mathrm{C}, \mathrm{D}$ ); (échelle $100 \mu$ : A, E) Annales de Parasitologie humaine et comparée (Paris), t. 47, $\mathrm{n}^{\circ} 3$ 
L'arrière cavité buccale est réduite à un épais collier chitinoïde à la base de la cavité buccale (cf. fig. I, B, C).

\section{DESCRIPTION DU MÂLE.}

Corps long de $8 \mathrm{~mm}$ sur $0,250 \mathrm{~mm}$ dans sa partie moyenne (toutes les dimensions données ci-après se rapportent à cet exemplaire). Capsule buccale haute de $120 \mu$, large au sommet de $125 \mu$ et à la base de $80 \mu$. Dimensions du trident médian : épine centrale longue de $90 \mu$, branches latérales longues de $100 \mu$. Cuticule épaisse de $5 \mu$, interstries égales à $4 \mu$. Anneau nerveux, deirides et pore excréteur respectivement situés à $225 \mu$, $280 \mu$ et $400 \mu$ de l'extrémité antérieure. Esophage musculaire et œsophage glandulaire respectivement longs de $500 \mu$ (largeur de $60 \mu$ à $120 \mu$ ) et $950 \mu$; longueur totale de l'œsophage égale à $1450 \mu$ (cf. fig. I, A).

Queue légèrement incurvée ventralement, à terminaison arrondie, sans pointe caudale. Cloaque à $150 \mu$ de l'extrémité postérieure. Ailes caudales moyennement développées, élargies à leur extrémité antérieure qui est située à $825 \mu$ de l'extrémité caudale (cf. fig. II, A, B). 2 spicules étroits, sinueux et effilés, inégaux. Spicule droit long de $625 \mu$, bien chitinisé ; spicule gauche long de $440 \mu$, faiblement chitinisé d'où son apparence plus grêle rendant son observation difficile sans dissection (cf. fig. II, E, F). Absence de gubernaculum.

La queue porte 15 paires de papilles caudales disposées comme suit (cf. fig. II, A, $\mathrm{B}, \mathrm{C}, \mathrm{D})$ :

- 7 paires de papilles précloacales finement pédonculées,

- 2 paires de papilles péricloacales également pédonculées mais plus trapues,

- 6 paires de petites papilles postcloacales, soit un $1^{\mathrm{er}}$ groupe de 2 , un $2^{\circ}$ groupe de 3 dont une latérale et 1 paire isolée à mi-distance entre le groupe de 3 et l'extrémité caudale.

\section{DESCRIPTION DE LA FEMELLE.}

Corps long de $17 \mathrm{~mm}$ sur 0,650 $\mathrm{mm}$ de large vers le milieu du corps (toutes les dimensions suivantes se rapportent à cet exemplaire).

Capsule buccale haute de $130 \mu$, large au sommet de $150 \mu$ et à la base de $100 \mu$. Dimensions du trident: épine centrale longue de $120 \mu$, branches latérales longues de $130 \mu$. Cuticule épaisse de $8 \mu$, interstries égales à $5 \mu$. Anneau nerveux, deirides et pore excréteur respectivement situés à $300 \mu, 400 \mu$ et $550 \mu$ de l'extrémité antérieure. Eso-

Fig. 2. - Camallanus ctenopomae n. sp. A: queue du mâle, vue latérale. B: queue du mâle, vue ventrale. C: queue du mâle vue latérale, détail de l'extrémité postérieure. D : queue du mâle, vut ventrale, détail de l'extrémité postérieure. E: spicule droit disséqué. F: spicule gauche disséqué. G: vulve et partie antérieure de l'ovéjecteur. $\mathrm{H}$ : larve (échelle $50 \mu: \mathrm{C}, \mathrm{D}, \mathrm{E}, \mathrm{F}, \mathrm{G}, \mathrm{H}$ ) ; (échelle $100 \mathrm{u}: \mathrm{A}, \mathrm{B})$ 
CAMALLANUS CTENOPOMAE N. SP.

387

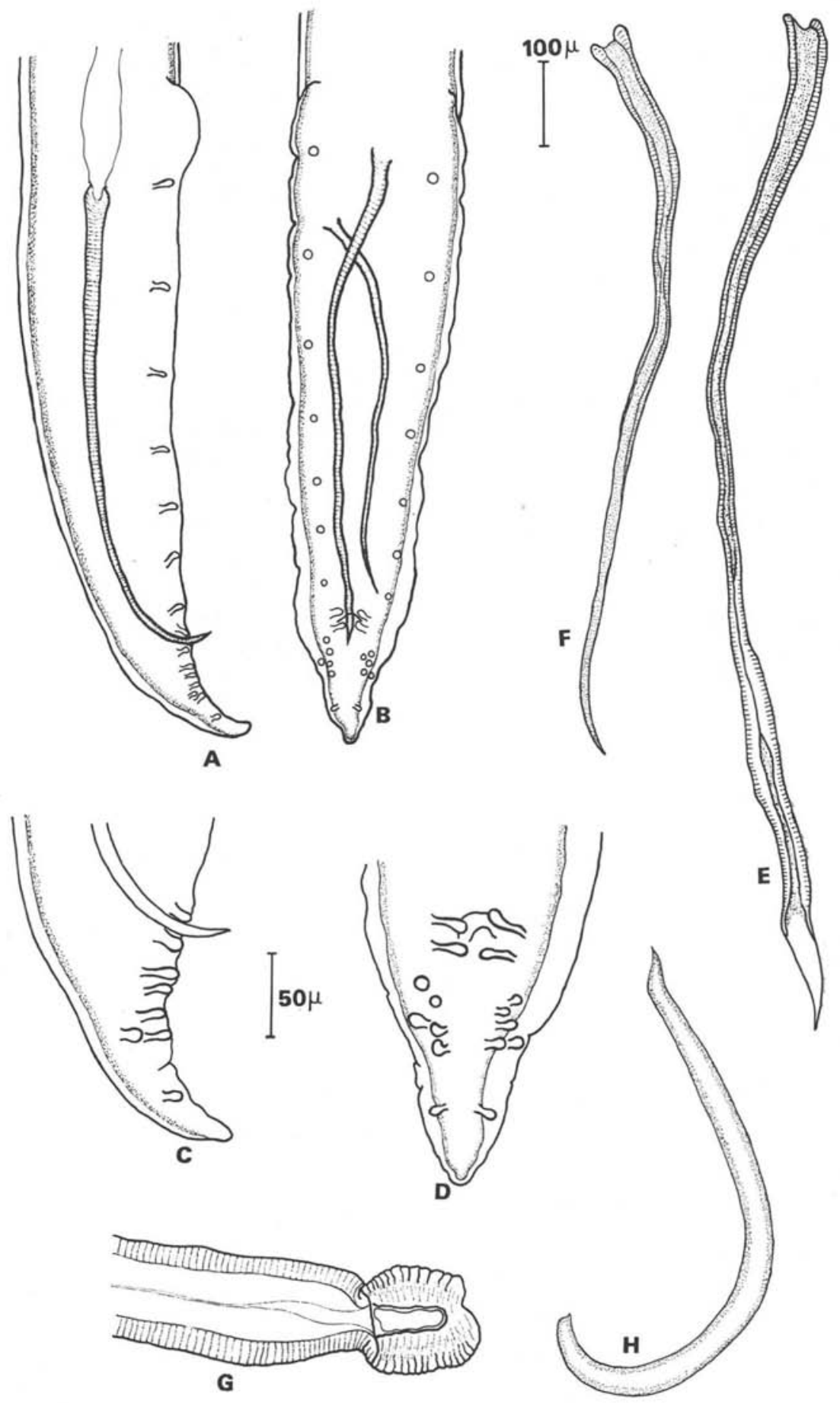


phage musculaire et œsophage glandulaire respectivement longs de $650 \mu$ (largeur de $100 \mu$ à $150 \mu$ ) et $1100 \mu$; longueur totale de l'œsophage égale à $1750 \mu$.

Extrémité caudale arrondie, sans pointe caudale. Anus à $325 \mu$ de l'extrémité postérieure (cf. fig. I, E).

Vivipare ; vulve située à $6,7 \mathrm{~mm}$ de l'apex, ovéjecteur musculaire dirigé vers l'arrière et long de $2000 \mu$ (cf. fig. II, G) ; 2 utérus à parois minces contenant un grand nombre de larves L 1 . Mensurations des larves au niveau de la vulve : $360 \mu$ de long sur $20 \mu$ de large (cf. fig. II, H).

\section{Discussion.}

Selon la classification proposée par Chabaud (in Grassé, 1965, p. 1032) les Nématodes que nous venons de décrire appartiennent au genre Camallanus Railliet et Henry, 1915 ; famille des Camallanidae Railliet et Henri, 1917, ordre des Spirurida ; notamment par les caractères de leur région buccale: capsule buccale formée par 2 valves latérales et pourvue de 2 tridents médians et absence de cavité buccale en arrière des valves.

Le genre Camallanus comprend à l'heure actuelle à notre connaissance, environ 35 espèces parasites de Vertébrés inférieurs, réparties sur tous les continents. Nous avons pu prendre connaissance de la description de toutes ces espèces à l'exception de $C$. salmonae Chakravorty, 1942, du Cachemire et C. sacconis $\mathrm{Li}, 1941$, de Chine, mais compte tenu de la localisation géographique de ces 2 espèces, il est peu probable qu'elles puissent être semblables à une espèce ouest-africaine du même groupe.

Parmi toutes les espèces étudiées, une dizaine seulement présentent des caractères morphologiques communs avec nos exemplaires et surtout : 7 à 11 crêtes denticulées sur chaque valve de la capsule buccale. Il s'agit de $C$. anabantis Pearse, 1933 chez Anabas testudineus et Clarias batrachus; C. unispiculus Khera, $1956 \mathrm{chez}$ Mastacembellus armatus ; C. nodulosus Gupta, 1959 chez Rana cynophlyctis ; C. thapari Gupta, 1959 chez Rana tigrina ; C. pearsei Yeh, $1960 \mathrm{chez}$ Rasbora daniconius ; C. kulasirii Yeh, 1960 chez Anabas testudineus et Ophicephalus punctatus ; C. fernandoi Yeh, $1960 \mathrm{chez}$ Ophicephalus punctatus et $O$. striatus ; C. spinosa Furtado, $1965 \mathrm{chez}$ Betta picta; C. mastacembeli Agrawal, 1967 chez Mastacembelus armatus et C. inglisi Agrawal, $1967 \mathrm{chez}$ Rana tigrina.

Toutes ces espèces présentent avec nos Camallanus des différences morphologiques notables. En effet, chez $C$. anabantis le mâle, qui ne mesure que $6,7 \mathrm{~mm}$, possède 2 spicules très inégaux $(590 \mu$ et $140 \mu)$, de plus les extrémités postérieures mâle et femelle portent 2 pointes caudales. Chez $C$. unispiculus la branche centrale des tridents médians est atrophiée et le mâle ne présente qu'un seul spicule. Chez $C$. nodulosus les crêtes buccales ne sont denticulées que dans la partie postérieure, l'épine médiane des tridents est courte, le spicule droit est armé d'une petite dent («prong») et mesure 440 à $450 \mu$, le spicule gauche mesure 270 à $360 \mu$. C. thapari présente également un spicule droit $(400$ à $460 \mu$ ) armé d'un éperon latéral de $40 \mu$, le spicule gauche mesure 280 à $320 \mu$. Chez 
C. pearsei la femelle est très petite $(5,1 \mathrm{~mm})$ avec seulement 7 crêtes denticulées sur chaque valve et pas de trident. Chez $C$. kulasirii les crêtes ne couvrent que les $2 / 3$ antérieurs des valves et les tridents sont complètement atrophiés. Chez $C$. fernandoi les crêtes ne portent qu'une seule dent, à leur base. $C$. spinosa est caractérisée par la présence de 4 rangées longitudinales d'épines cuticulaires. Chez $C$. mastacembeli le mâle mesure 11,6 à 12,64 mm et ne possède qu'un seul spicule long de $336 \mu$. Chez $C$. inglisi le spicule droit mesure 270 à $360 \mu$ et porte un éperon de $50 \mu$, le spicule gauche est long de 370 à $460 \mu$.

Il nous semble donc que notre espèce est nouvelle et nous proposons de la nommer Camallanus ctenopomae n. sp.

C'est la $2^{\circ}$ espèce du genre Camallanus connue en Afrique chez les Poissons d'eaı douce après C. kirandensis Baylis, 1928, du Tanganyika.

\section{Bibliographie}

Agrawal (V.), 1967. - Some new Camallanoidea (Spirurida). Nematodes from Fishes, Amphibians and Reptiles. Ann. Parasit., 42, 327-342.

BAYLIs (H. A.), 1928. - Some Parasitic Worms, mainly from Fishes, from Lake Tanganyika. Ann. Mag. Nat. Hist., ser. 10, vol. I, 552-562.

Furtado (J. I.), 1965. - Zeylanema spinosa sp. nov. (Camallanidae) from a Malayan freshwater fish. Ann. Parasit., 40, 677-680.

Grasse (P.-P.), 1965. — Traité de Zoologie. Némathelminthes, t. IV, fasc. III, 1813-2758, Masson et $\mathrm{C}^{10}$ Editeurs, Paris.

Gupta (S. P.), 1959. - Nematode parasites of Vertebrates of East Pakistan. III. Camallanidae from Fish, Amphibia and Reptiles. Can. J. Zool., 37, 771-779.

KHERA (S.), 1956. - Nematode parasites of some Indian Vertebrates. Indian J. Helminth., 6, 27-133.

Pearse (A. S.), 1933. - Parasites of Siamese fishes and Crustaceans. J. Siam. Soc., (nat. hist. suppl.), 9, 179-191.

YEH (L. S.), 1960. - On a collection of Camallanid Nematodes from Freshwater Fishes in Ceylon. J. Helminth., 34, 107-116. 